\title{
Shadow Fading Cross-Correlation of Multi-Frequencies in Curved Subway Tunnels
}

\author{
Bei Zhang , Zhangdui Zhong , Ke Guan , Ruisi He , and Cesar Briso-Rodríguez \\ State Key Laboratory of Rail Traffic Control and Safety, Beijing Jiaotong University, Beijing, China \\ ETSI y Sistemas de Telecomunicación. Technical University of Madrid, 28031, \\ Madrid, Spain \\ E-mail: zbandysun@gmail.com
}

\begin{abstract}
Radio propagation characteristics in curved tunnels are important for designing reliable communications in subway systems. In this paper, shadow fading is characterized, and cross-correlation property of shadow fading for different frequency bands is investigated based on empirical measurements. The measurements were conducted in two types of curved subway tunnels with $300 \mathrm{~m}$ and $500 \mathrm{~m}$ radii of curvatures at $980 \mathrm{MHz}, 2400 \mathrm{MHz}$, and $5705 \mathrm{MHz}$, respectively. The impact of antenna polarization and propagation environment on shadow fading correlation at the receiver is evaluated. It is found that shadow fading with horizontal polarized antenna exhibits less correlation than with vertical polarized antenna. Strong independence of shadowing correlation and tunnel type is observed. Furthermore, a heuristic explanation of the particular shadowing correlation property in subway tunnel is presented.
\end{abstract}

\section{INTRODUCTION}

Intelligent transportation system (ITS) integrates information and communication technology exploiting the existing technologies/infrastructures, such as computers, communication networks, sensors, positioning systems, and automation technologies for collecting relevant data [1]. The subway system is an indispensable part of the ITS. Various kinds of wireless communication technologies proposed for metropolitan subway system play a significant role to ensure personnel safety, enhance operational efficiency and process optimization. More importantly, reliable communication technologies in subways that incorporate safety and navigational/infotainment features as well as positive environmental impact are regarded as basic requirements for the sustainable wealth and prosperity of modern societies.

There are many investigations of propagation channels in tunnel scenarios [2-6]. The propagation characteristics in circular tunnels at $450 \mathrm{MHz}$ and $900 \mathrm{MHz}$, arched railway and road tunnels at $400 \mathrm{MHz}$, subway tunnels at $2.4 \mathrm{GHz}$, as well as $5.8 \mathrm{GHz}$ are studied, respectively. Authors in [7] investigate the propagation of electromagnetic waves through various media, and the channel model for underground mines, roads, and subway tunnels are proposed. It is demonstrated in [8] that tunnels with rectangular and circular cross sections can be explained via modal effects. However, radio propagation in curved subway tunnels, whose cross-section size is much smaller than road, mine, or railway tunnels, have been rarely addressed in previous literatures.
A clear comprehension of the large-scale fading that characterizes the radio signal attenuation over long transmission distance is crucial in future wireless network planning. In other words, link budget in reality is a very important piece of network planning, and it requires a characterization of shadow fading. Shadow fading describes the loss caused by obstacles along the propagation path. It is widely accepted that the shadow fading follows a log-normal distribution. Shadow fading has been widely investigated in railway and vehicular networks [9-11]. Since shadow fading is determined by surrounding buildings, which are usually similar to adjacent links, the shadow fading components from different communication links are mostly correlated. Study of the correlation property of shadow fading plays a key role in designing handover algorithms, evaluating performances of macro-diversity schemes, and significantly affects interference power which consequently perturbs the system performance [12]. Most of the current channel models assume that different channels from one mobile station (MS) to several base stations (BSs) are independent. In the wireless world initiative new radio (WINNER) models as well as in spatial channel models (SCM), shadow fading cross-correlation is characterized by a correlation coefficient of 0.5 [13]. However, little attention has been paid to cross-correlation property from different links operating at different frequency bands in previous research. In [14], correlation between shadowing in the GSM-900 and the GSM-1800 band is investigated. The authors in [15] analyze the correlation of shadow fading in a suburban environment at different frequency bands. To the best of the authors' knowledge, no other paper has addressed the multi-frequency shadow fading correlation, especially in the curved subway tunnel.

In this paper, we investigate the shadow fading crosscorrelation property for different frequency bands and the impact of environmental system parameters in curved tunnels of subways. The results would be useful in the communication system design: if shadow fading is found to be uncorrelated among different frequency bands in tunnel scenario, one might use frequency diversity to maintain a reliable communication link between the transmitter and the receiver in emergency cases; it is of interest to determine whether propagation channels in different frequency bands are correlated or not, which helps to develop a channel 
simulator.

The remainder of the paper is organized as follows. Section II describes measurement campaign in subway tunnels in Spain. In section III, shadow fading distribution as well as the correlation coefficient is discussed. Section IV depicts the experimental investigation results in some tables and corresponding heuristic explanations are presented. Conclusions are drawn in section $\mathrm{V}$.

\section{Measurement Summary}

The narrowband measurements were taken along arched curved tunnels in the subway of Madrid, Spain. There are two types of arched tunnels in the modern subway systems, named Arched "Type I" and Arched "Type II" as mentioned with more details in [16] [17]. Arched "Type I" tunnel is composed of three plane walls and an arched roof with dimensions $7.59 \times 5.52 \mathrm{~m}$. Arched "Type II" tunnel includes arched walls and a plane floor roof with dimensions $8.41 \times 6.87 \mathrm{~m}$, which is more similar to a semicircular shape than "Type I". The tunnel shape and measurement scenario are outlined in Fig. 1. In total, over $4 \mathrm{~km}$ of measurements were taken, with one measurement activated at least every $0.5 \mathrm{~m}$. Along the routes, the links between BSs and MS experienced line-of-sight (LOS) and non lineof-sight (NLOS) conditions.

Measurement data were collected at popular frequency bands in public communication systems or signaling and train control systems deployed in subway systems, i.e., 980 $\mathrm{MHz}, 2400 \mathrm{MHz}$, and $5705 \mathrm{MHz}$. The sampling intervals were shorter than one wavelength. We used continuous wave (CW) at transmitters (Tx) with panel antennas. The transmit antennas were horizontal polarization directional and vertical polarization directional with the gain of $14 \mathrm{dBi}, 8 \mathrm{dBi}$, and $19 \mathrm{dBi}$, respectively. Output power of Tx was $27 \mathrm{dBm}, 22$ $\mathrm{dBm}$, and $24 \mathrm{dBm}$ for $980 \mathrm{MHz}, 2400 \mathrm{MHz}$, and 5705 $\mathrm{MHz}$, respectively. The receiver $(\mathrm{Rx})$ consisted of a receiver antenna, a lower noise amplifier, and a spectrum analyzer which was connected to a laptop for capturing received signal power for further processing. The omnidirectional receiver antenna was a directive $8.5 \mathrm{dBi}$ gain logperiodic antenna, attached to the front windshield of standard metro train with the height of $3 \mathrm{~m}$ above the ground. Tx were positioned 4.5 $m$ away from the train. The relative position of $T x$ and $R x$ along the train route are also illustrated in Fig. 1.

In order to observe the impact of some system parameters on multi-frequency shadowing correlation, namely the antenna polarization, the radius of curvature, and arched tunnel type, the propagation measurements were carried out in these two types of curved subway tunnels with different configurations.

It has to point out that for our system, sampling of the receiver via distance sensor does not change with the speed of the train. Therefore, the spatial sampling interval is not constant, i.e., the distance between two samples from differen sets is unequal, but the difference is not substantial. We consider four factors in our analysis: cross section of tunnel, radius of curvature, polarization, and frequency. However,
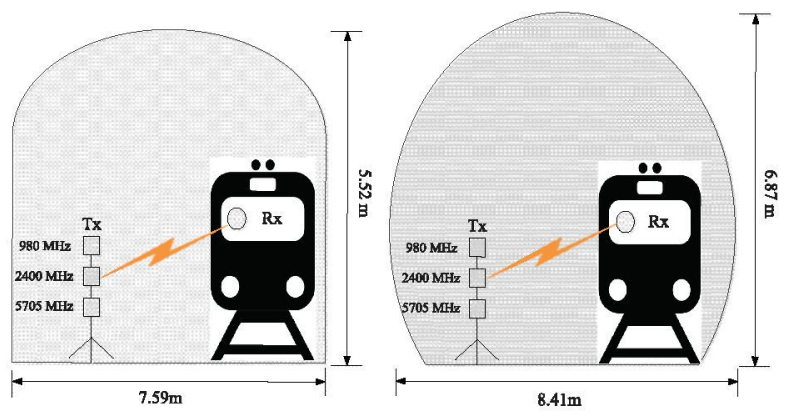

Fig. 1. Measurement schematic diagram of different arched tunnels.

not all the combinations are covered due to the limited conditions of the measurements in reality. Therefore, a model for shadow fading correlation is not proposed due to limited dataset. Instead, the cross-correlation is characterized in this paper.

\section{ANALYSIS OF MEASUREMENT DATA}

\section{A. Shadow Fading Distribution}

In subway systems, the received power can be shadowed due to the presence of obstructions such as a large number of cables, fire facilities. To extract the shadow fading component, the distance-dependent path loss is removed from the received power (in dB) using linear regression fit [18] [19]. Shadowing gets worse with an increase of carrier frequency. The effect of small-scale fading is removed by averaging using a $40 \lambda$ window as suggested by [20], and the averaged received power level can be expressed as

$$
p(d)=P_{0}-n \cdot \log _{10}\left(\frac{d}{d_{0}}\right)+S F(d),
$$

where $d$ is the distance between Tx and $\mathrm{Rx}, n$ is the path loss exponent, and $P_{0}$ is a reference value at the reference distance $d_{0}$. To extract the shadow fading components, the distance dependent path loss is removed from the received power (in $\mathrm{dB}$ ) via linear regression fit.

The received power without small-scale fading under different operating frequency bands is shown in Fig. 2, which is acquired in "Type I" tunnel. It can be observed that the signal at $980 \mathrm{MHz}$ is the strongest, due to the smallest propagation loss at low frequency band and large output power in the measurements. It is also noteworthy that the large-scale fluctuation of received power behaves in a similar way for the results with different frequency bands, which implies a cross-correlation for the signals between two frequency bands can be extracted (discussed later).

Numerous empirical results demonstrate that the shadow fading can be modeled as a log-normal random variable. In other words, the shadow fading behaves as a Gaussian random variable in the $\mathrm{dB}$ scale. Thus, the probability density function (PDF) for the power of shadow fading (SF) is given by

$$
p_{S F}(s)=\frac{\xi}{\sqrt{2 \pi} s \sigma_{s}} \exp \left[-\frac{\left(10 \log _{10} s-\mu_{s}\right)^{2}}{2 \sigma_{s}^{2}}\right]
$$




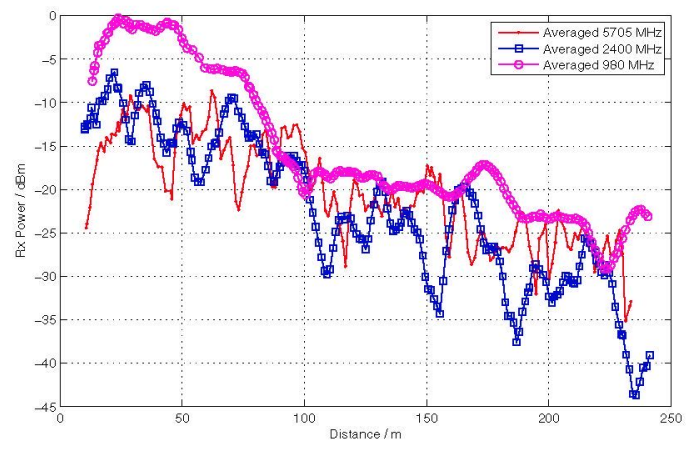

Fig. 2. Averaged power as a function of distance from BS.
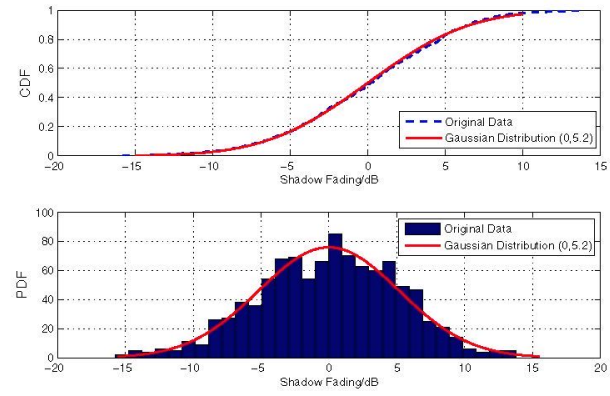

Fig. 3, CDF and PDF of the measured shadow fading, together with a Gaussian fit at $5705 \mathrm{MHz}$.

where $\xi=10 / \ln 10, \mu_{s}$ is the mean of $10 \log _{10}(s)$ in $\mathrm{dB}$, and $\sigma_{s}$ is the standard deviation of shadow fading, also in dB. Typical value of $\sigma_{s}$ are 4 to $10 \mathrm{~dB}$ [26]. Fig. 3 presents the cumulative distribution function (CDF) and the PDF of shadow fading component extracted from empirical received signals at $5705 \mathrm{MHz}$. It indicates that the shadow fading component follows a log-normal distribution (or Gaussian in $\mathrm{dB}$ scale) in curved tunnel scenario, which is coincided with the measurement results reported in [14]. The standard deviation of the shadow fading in Fig. 3 is $5.2 \mathrm{~dB}$, and the standard deviation of the whole measurements changes from 2.7 to $6.5 \mathrm{~dB}$. Moreover, most of them vary from 3 to $5 \mathrm{~dB}$. In comparison, the standard deviation of shadow fading in different scenarios is illustrated according to [25] as shown in Table I.

\section{B. Correlation of Shadow Fading for Multi-frequency Bands}

Shadow fading correlations are mostly caused by some similar scatters contributing to different links (similarity of the environment). From the perspective of downlink in wireless systems, two kinds of shadow fading correlations can be defined as shown in Fig. 4, which are similar to [25]:

1) Transmit Correlation: the statistical correlation between the signals received by two separate MSs at two different locations, from the same BS.

2) Receive Correlation: the statistical correlation between the signals originating from two disparate BSs and arriving at the same MS.
It is restricted to receive correlation in this paper, i.e., shadowing cross-correlation between one single MS and different BSs operating at $980 \mathrm{MHz}, 2400 \mathrm{MHz}$, and $5705 \mathrm{MHz}$ frequency bands, respectively. The shadow fading crosscorrelation coefficient $\rho$ is defined as the cross-correlation coefficient between shadow fading components of the signals received from two BSs for different frequency bands, which can be evaluated using

$$
\rho=\frac{\sum_{i=1}^{n}\left(X_{i}-\bar{X}\right)\left(Y_{i}-\bar{Y}\right)}{\sqrt{\sum_{i=1}^{n}\left(X_{i}-\bar{X}\right)^{2}} \sqrt{\sum_{i=1}^{n}\left(Y_{i}-\bar{Y}\right)^{2}}},
$$

where $X_{i}$ and $Y_{i}$ denote the shadow fading components of two separate received power, $\bar{X}$ and $\bar{Y}$ are the mean of shadow fading components, $n$ is the number of the shadow fading component samples obtained at the same receiver. Here, $n$ ranges from 200 to 400 in different measurement routes according to the decorrelation distance. If the absolute value of the estimated correlation coefficient lies in $[0,0.1]$, two random variables can be treated as uncorrelated.

\section{RESULTS AND DisCUSSIONS}

We evaluate the absolute value of shadowing receive correlation coefficients for multi-frequency bands, and discuss the impact of antenna polarization and propagation environments (including the radius and the type of curved tunnel). The results are summarized in Table II, Table III, and Table IV.

Table II lists cross-correlation coefficients with vertical and horizontal polarizations. The horizontal polarization tends to have a lower cross-correlation than the vertical polarization. In addition, it is observed that as the operating frequency increases, the cross-correlation reduces.

The cross-correlation coefficients with different radius of curvature are shown in Table III. The shadowing crosscorrelation with smaller curved radius tends to be weaker, but the difference is not substantial. It indicates that the variation of curved radius in the tunnel has little impact on shadowing cross-correlation.

For comparison, the shadow fading correlation between $0.9 \mathrm{GHz}, 1.8 \mathrm{GHz}$, and $2.1 \mathrm{GHz}$ bands respectively is found to range from 0.7 to 0.84 in suburban environment [15], where it is suggested that shadowing, which is mainly caused by blockage from obstacles, behaves in a similar way for all frequency bands and causes similar attenuation for all frequencies in suburban environment. Compared with the results in [15], it can be concluded that the crosscorrelation of shadow fading for different frequency bands in curved tunnel environments is weaker than that in suburban environments. A heuristic explanation is as follows:

1) According to electromagnetic modal theory, the radio propagation in tunnels consists of four propagation zones [21-23], namely: free-space propagation zone, multi-mode propagation zone, fundamental mode propagation zone, and extreme far zone. The point of transition from multi-mode propagation to fundamental mode propagation zone is called 

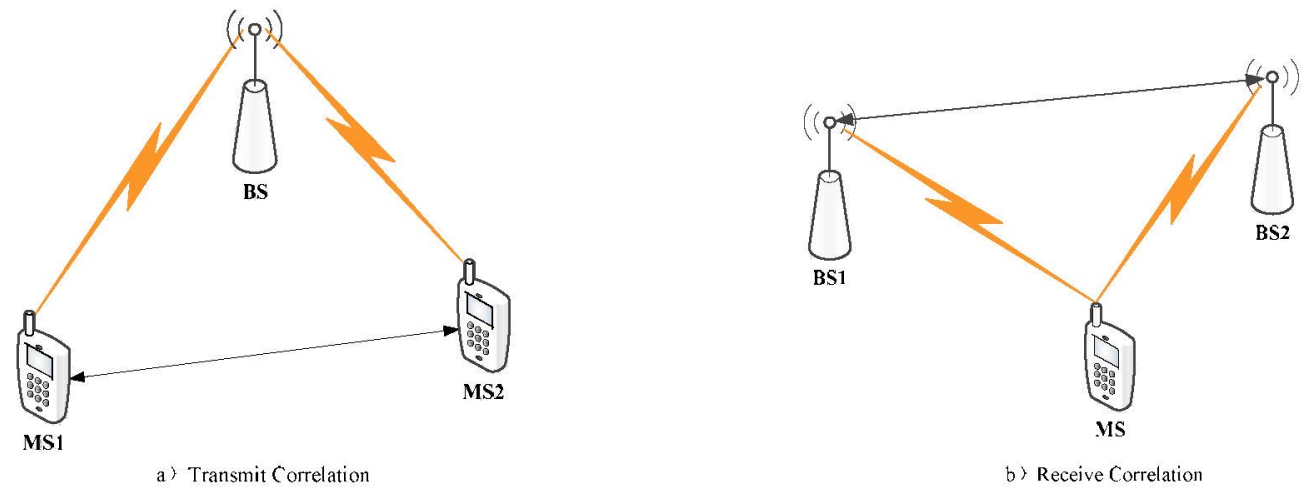

Fig. 4. Different types of shadowing cross-correlation with fixed BS and fixed MS.

TABLE I

A REFERENCE OF STANDARD DEVIATION OF SHADOW FADING FOR DIFFERENT SCENARIOS

\begin{tabular}{|c|c|c|c|c|}
\hline Scenario & Definition & Mob. $\mathrm{km} / \mathrm{h}$ & Frequency $(\mathrm{GHz})$ & Standard deviation (LOS/NLOS) \\
\hline A1 & Indoor office & $0-5$ & $2-6$ & $3 / 4$ \\
B1 & Typical urban micro-cell & $0-5$ & $2-6$ & $3 / 4$ \\
B3 & Large indoor hall & $0-5$ & $2-6$ & $3 / 4$ \\
C1 & Suburban & $0-5$ & $2-6$ & $4(6) / 8$ \\
C2 & Typical urban macro-cell & $0-5$ & $2-6$ & $4(6) / 8$ \\
D1 & Rural macro-cell & $0-5$ & $2-6$ & $4(6) / 8$ \\
D2a & Moving netwroks & $0-350$ & $2-6$ & 4 \\
\hline
\end{tabular}

TABLE II

SHADOWING CROSS-CORRELATION COEFFICIENTS WITH DIFFERENT POLARIZATION MODES

\begin{tabular}{|c|c|c|c|c|}
\hline \multicolumn{3}{|c}{ Tunnel: Arched "Type I" } & \multicolumn{2}{c|}{ Radius of Curvature: $500 \mathrm{~m}$} \\
\hline Polarization & \multicolumn{2}{|c|}{ Vertical Polarization } & Horizontal Polarization \\
\hline Frequency & $980 \mathrm{MHz}$ vs $2400 \mathrm{MHz}$ & 0.41 & $980 \mathrm{MHz}$ vs $2400 \mathrm{MHz}$ & 0.24 \\
\hline Frequency & $980 \mathrm{MHz}$ vs $5705 \mathrm{MHz}$ & 0.24 & $980 \mathrm{MHz}$ vs $5705 \mathrm{MHz}$ & 0.13 \\
\hline Frequency & $2400 \mathrm{MHz}$ vs $5705 \mathrm{MHz}$ & 0.077 & $2400 \mathrm{MHz}$ vs $5705 \mathrm{MHz}$ & 0.081 \\
\hline
\end{tabular}

TABLE III

SHADOWING CROSS-CORRELATION COEFFICIENTS WITH DIFFERENT RADII OF CURVATURE

\begin{tabular}{|c|c|c|c|c|}
\hline \multicolumn{2}{|c|}{ Tunnel: Arched "Type II" } & \multicolumn{2}{c|}{ Polarization: Horizontal } \\
\hline Radius of Curvature & \multicolumn{2}{|c|}{$\mathrm{R}=300 \mathrm{~m}$} & \multicolumn{2}{c|}{$\mathrm{m} 00 \mathrm{~m}$} \\
\hline Frequency & $980 \mathrm{MHz}$ vs $2400 \mathrm{MHz}$ & 0.23 & $980 \mathrm{MHz}$ vs $2400 \mathrm{MHz}$ & 0.27 \\
\hline Frequency & $980 \mathrm{MHz}$ vs $5705 \mathrm{MHz}$ & 0.12 & $980 \mathrm{MHz}$ vs $5705 \mathrm{MHz}$ & 0.041 \\
\hline Frequency & $2400 \mathrm{MHz}$ vs $5705 \mathrm{MHz}$ & 0.039 & $2400 \mathrm{MHz}$ vs $5705 \mathrm{MHz}$ & 0.073 \\
\hline
\end{tabular}

the dividing point 2, namely "break point". It is localized at the distance where the second fundamental modes have suffered one reflection from vertical or horizontal walls. The distance from Tx to dividing point 2 can be expressed by [23]

$$
D_{2}(\lambda) \approx \max \left(\frac{W^{2}}{\lambda}, \frac{H^{2}}{\lambda}\right)
$$

where $D_{2}$ signifies the position of the second dividing point, the width and height of the equivalent rectangular tunnel given by $W$ and $H$, and $\lambda$ denotes the wavelength. Therefore, the distance from the Tx to the "break point" for $980 \mathrm{MHz}$, $2400 \mathrm{MHz}$, and $5705 \mathrm{MHz}$ are approximately equal to 231.1 $\mathrm{m}, 565.8 \mathrm{~m}$, and $1345.1 \mathrm{~m}$, respectively. Given that condition, the received power fluctuation is dominated by different modal effects because the sampling start point is usually about $100 \mathrm{~m}$ of $\mathrm{Tx}-\mathrm{Rx}$ during the calculation of shadowing cross-correlation coefficients. It is also proposed that the break point is smaller in the case of a curved tunnel than for a straight tunnel in [24].

2) The analytical expression of the propagation loss in the multimode propagation zone $L_{M M}$ is [23]

$$
\begin{aligned}
& L_{M M}(d B) \\
& =10 \log _{10}\left[\sum_{i=1}^{m} \sum_{j=1}^{n} \sqrt{10^{2 \alpha(i, j)^{h}}\left|z_{r}-z_{t}\right|+10^{2 \alpha(i, j)^{v}}\left|z_{r}-z_{t}\right|}\right] \\
& \quad+L_{w}\left(\left|z_{r}-z_{t}\right|\right),
\end{aligned}
$$

where $\left|z_{r}-z_{t}\right|$ denotes the distance between Tx and $\mathrm{Rx} ; L_{w}$ denotes the loss owing to the roughness of the walls [27]. The attenuation constants $\alpha(i, j)^{h}, \alpha(i, j)^{v}$ are given by [28],

$$
\alpha(i, j)^{h}=4.343 \lambda^{2}\left(\frac{m^{2} \varepsilon_{v}}{a^{3} \sqrt{\varepsilon_{v}-1}}+\frac{n^{2}}{b^{3} \sqrt{\varepsilon_{h}-1}}\right),
$$


TABLE IV

SHADOWING CROSS-CORRELATION COEFFICIENTS WITH DIFFERENT TYPES OF CURVED TUNNELS

\begin{tabular}{|c|c|c|}
\hline \multicolumn{2}{|c|}{ Radius of Curvature: $500 \mathrm{~m}$} & Polarization: Horizontal \\
\hline $980 \mathrm{MHz}$ & $2400 \mathrm{MHz}$ & $5705 \mathrm{MHz}$ \\
\hline 0.049 & 0.051 & 0.037 \\
\hline
\end{tabular}

$$
\alpha(i, j)^{v}=4.343 \lambda^{2}\left(\frac{m^{2} \varepsilon_{v}}{a^{3} \sqrt{\varepsilon_{v}-1}}+\frac{n^{2} \varepsilon_{h}}{b^{3} \sqrt{\varepsilon_{h}-1}}\right),
$$

where $\varepsilon_{v}$ and $\varepsilon_{h}$ are the relative permittivities of the vertical and horizontal walls, $a$ and $b$ are the horizontal and vertical dimensions of the tunnel. Therefore, an increase in frequency results in more loss for the effect of multi-mode in waveguides. Moreover, the field of radio waves $E_{m n}$ at the position $\left(x, y,\left|z_{r}-z_{t}\right|\right)$ in tunnels can be expressed by summing up the field of limited excited modes [29]:

$$
\begin{aligned}
& E^{R x}\left(x, y,\left|z_{r}-z_{t}\right|\right)= \\
& \left\lfloor\frac{W}{H} \sqrt{4 H^{2} f_{0}^{2} \mu_{0} \varepsilon_{0} \varepsilon_{a}-1}\right\rfloor\left\lfloor\frac{H}{W} \sqrt{4 W^{2} f_{0}^{2} \mu_{0} \varepsilon_{0} \varepsilon_{\alpha}-m^{2}}\right\rfloor \\
& \sum_{\substack{m=1 \\
\rho_{m n}}}^{E_{m, n}^{\text {eign }}}(x, y) \cdot e^{-\left(\alpha_{m n}+i \beta_{m n}\right) \cdot\left|z_{r}-z_{t}\right|}
\end{aligned}
$$

where $\varepsilon_{0}$ is the relative permittivity in vacuum space, $\varepsilon_{a}$ is the relative permittivity for the air in the tunnel, and $\rho_{m n}$ is the mode intensity on the excitation plane; $\alpha_{m n}$ and $\beta_{m n}$ are the attenuation coefficient and the phase-shift coefficient, respectively, given by

$$
\begin{gathered}
\alpha_{m n}=\frac{2}{W}\left(\frac{m \pi}{W k}\right)^{2} \operatorname{Re} \frac{\varepsilon_{r v}^{*}}{\sqrt{\varepsilon_{r v}^{*}-1}}+\frac{2}{H}\left(\frac{n \pi}{H k}\right)^{2} \operatorname{Re} \frac{1}{\sqrt{\varepsilon_{r h}^{*}-1}} \\
\beta_{m n}=\sqrt{k^{2}-\left(\frac{m \pi}{W}\right)^{2}-\left(\frac{n \pi}{H}\right)^{2}},
\end{gathered}
$$

where $\varepsilon_{r v}^{*}, \varepsilon_{r h}^{*}$ are the relative electrical parameters. It can be inferred that the shadow fading in tunnel environments is also caused by the phase-shift. Hence, the correlation property of shadow fading in tunnel environments may behave differently with that in suburban environments.

3) The energy impinging on the tunnel walls does not only undergo specular reflection but it is on the contrary spread in many different directions due to the surface roughness. The wall roughness may lead to extra shadowing loss of received power [30-32].

It is worth mentioning that correlation coefficients typically range from -0.1 to 0.1 at $2400 \mathrm{MHz}$ vs $5705 \mathrm{MHz}$, which means that the shadowing cross-correlation is nearly uncorrelated. Because uncorrelated Gaussian random variables are independent as well as shadow fading is assumed as lognormal distribution, it can be concluded that wireless network planning in tunnel scenario can be conducted under the assumption that shadow fading at $2400 \mathrm{MHz}$ and $5705 \mathrm{MHz}$ are independent. For this reason, frequency diversity at these frequency bands may be considered as a suitable solution to combat shadow fading to ensure reliable communication in subway systems for emergency cases.

In order to investigate the impact of propagation environment, the shadowing correlation at the same frequency are computed as shown in Table IV, there is almost no shadowing correlation relation exist between different types of tunnel at $980 \mathrm{MHz}, 2400 \mathrm{MHz}$, and $5705 \mathrm{MHz}$, in other words, they are uncorrelated with each other. It indicates that the type of tunnel can significantly affect the shadow fading of received signal. As a consequence, it is suggested that the network planning in different types of curved tunnels should be conducted independently.

\section{CONCLUSION}

This paper has investigated cross-correlation property of shadow fading in curved tunnels. A measurement campaign has been conducted to measure the propagation channel in $980 \mathrm{MHz}, 2400 \mathrm{MHz}$ and $5705 \mathrm{MHz}$ simultaneously. Results confirm that shadowing correlation characteristics depend jointly on antenna polarization and propagation environments. Typical standard deviations of log-normal shadow fading in tunnel environments is found to be $3 \sim 4 \mathrm{~dB}$. Frequency diversity of $2400 \mathrm{MHz}$ and $5705 \mathrm{MHz}$ is feasible in subway systems. A heuristic explanation of the shadowing cross-correlation property is presented. The reasons for lower shadowing cross-correlation in tunnel environments is conjectured to be the specific propagation behaviors at different frequency bands according to modal theory.

\section{ACKNOWLEDGMENT}

This work is supported by State Key Laboratory of Rail Traffic Control and Safety Project (RCS2014ZT32), Beijing Jiaotong University, the Fundamental Research Funds for the Central Universities (No. 2014JBZ021), the National 863 Project Granted No. 2014AA01A706, the Key grant Project of Chinese Ministry of Education (No.313006), and the National Natural Science Foundation of China under Grant No. U1334202. This work has also being developed under the framework of INNPACTO TECRAIL research project IPT-2011-1034-37000 funded by the Spanish Ministry of Economy and Competitiveness.

\section{REFERENCES}

[1] R. Gulzaib, et al., "What's new in intelligent transportation systems," Vehicular Technology Magazine, IEEE, vol.8, pp. 45- 69, Dec. 2013.

[2] D. Dudley, M. Lienard, S. Mahmoud, and P. Degauque, "Wireless propagation in tunnels," IEEE Antenna PropagatMag., vol. 49, no. 2, pp. 11-26, 2007.

[3] A. Hrovat, G. Kandus, and T. Javornik, "Four-slope channel model for path loss prediction in tunnels at $400 \mathrm{MHz}, "$ Microwaves, Antennas and Propagation, IET, vol. 4, no. 5, pp. 571-582, 2010.

[4] R. He, Z. Zhong, and C. Briso, "Broadband channel long delay cluster measurements and analysis at $2.4 \mathrm{GHz}$ in subway tunnels," in Proc. IEEE 73rd Veh. Technol. Conf. (VTC), Hungary, 2011, pp. 1-5.

[5] R. He, Z. Zhong, B. Ai, K. Guan, B. Chen, J. AIonso, and C. Briso, "Propagation channel measurements and analysis at $2.4 \mathrm{GHz}$ in subway tunnels," Microwaves, Antennas and Propagation, IET, vol. 7, no. 11, pp. 934-941, 2013. 
[6] J. Alonso, S. Capdevila, B. Izquierdo, and J. Romeu, "Propagation measurements and simulations in tunnel environment at $5.8 \mathrm{GHz}$," in Antennas and Propagation Society International Symposium, 2008. APS 2008. IEEE, pp. 1-4, 2008.

[7] I. F. Akyildiz, Z. Sun, and M. C. Vuran, "Signal propagation techniques for wireless underground communication networks," Physical Communication Journal, vol. 2, no. 3, pp. 167-183, Sept. 2009.

[8] S. Mahmoud, "On modal propagation of high frequency electromagnetic waves in straight and curved tunnels," in Antennas and Propagation Society International Symposium, 2004. IEEE, vol. 3, pp. 2963-2966, 2004

[9] R. He, Z. Zhong, B. Ai, and J. Ding, "Propagation measurements and analysis for high-speed railway cutting scenario," Electron. Lett., vol. 47, no. 21, pp. 1167-1168, Oct. 2011.

[10] R. He, Z, Zhong, B. Ai, G. Wang, J. Ding, and A. F. Molisch, "Measurements and analysis of propagation channels in high-speed railway viaducts," IEEE Trans. Wireless Commun., vol. 12, no. 2, pp. 794-805, 2013.

[11] R. He, A. F. Molisch, F. Tufvesson, Z. Zhong, B. Ai, and T. Zhang, Vehicle-to-vehicle propagation models with large vehicle obstructions, IEEE Transactions on Intelligent Transportation Systems, pp. 1-12, 2014.

[12] S. S. Szyszkowicz, H. Yanikomeroglu, J. S. Thompson, "On the feasibility of wireless shadowing correlation models," IEEE Trans. Veh. Technol., vol, 59, no. 9, pp. 4222-4236, Nov. 2010.

[13] D. S. Baum, et al., "Final Report on Link Level and System Level Channel Models," IST-2003-507581 WINNER D5. 4, Nov. 2005.

[14] P. E. Mogensen, P. Eggers, C. Jensen, and J. B. Andersen, "Urban area radio propagation measurements at 955 and $1845 \mathrm{MHz}$ for small and micro cells, in Proceedings of Global Telecommunications Conference (GLOBECOM), vol. 2, pp. 1297-1302, Dec. 1991.

[15] C. Van Laethem, F. Quitin, F. Bellens, C. Oestges, and P. De Doncker, "Correlation for multi-frequency propagation in urban environments," Progress in Electromagnetics Research Lett., vol. 29, pp. 151-156, 2012.

[16] K. Guan, Z. Zhong, B. Ai, R. He, Y. Li, and C. Briso, "Propagation mechanism modeling in the near-region of arbitrary cross-sectional tunnels," International Journal of Antennas and Propag., vol. 2012, pp. 11, 2012, Art. ID 183145 .

[17] K. Guan, Z. Zhong, B. Ai, and C. Briso, "Modeling of the division point of different propagation mechanisms in the near-region within arched tunnels," Wireless Personal Communications, vol. 68, no. 3, pp. 489-505, 2013. [Online]. Available: http://dx.doi.org/10.1007/s11277011-0464-7.

[18] R. He, Z. Zhong, B. Ai, J. Ding, and K. Guan, "Analysis of the relation between Fresnel zone and path loss exponent based on two-ray model," IEEE Antennas Wireless Propag. Lett., vol. 11, pp. 208-211, 2012.

[19] R. He, Z. Zhong, B. Ai, and J. Ding, "An empirical path loss model and fading analysis for high-speed railway viaduct scenarios," IEEE Antennas Wireless Propag. Lett., vol. 10, pp. 808-812, 2011.

[20] W. C. Y. Lee, "Estimate of local average power of a mobile radio signal," IEEE Trans. Veh. Technol., vol. 34, no. 1, pp. 22-27, Feb. 1985.

[21] K. Guan, Z. Zhong, B. Ai, R. He, and C. Briso, "Five-Zone Propagation Model for Large-Size Vehicles inside Tunnels," Progress In Electromagnetics Research, vol. 138, pp. 389-405, 2013.

[22] K. Guan, Z. Zhong, B. Ai, R. He, B. Chen, Y, Li, and C. Briso, "Complete Propagation Model Structure inside Tunnels," Progress In Electromagnetics Research, vol. 141, pp. 711-726, 2013.

[23] K. Guan, Z. Zhong, B. Ai, R. He, B. Chen, Y, Li, and C. Briso, "Complete propagation modeling in tunnels," IEEE Antennas Wireless Propag. Lett., vol. 12, pp. 741C744, Jun. 2013.

[24] E. Masson, Y. Cocheril, P. Combeau, L. Aveneau, M. Berbineau, R. Vauzelle, and E. Fayt, "Radio wave propagation in curved rectangular tunnels at $5.8 \mathrm{GHz}$ for metro applications," in ITS Telecommunications (ITST), 2011 11th International Conference on, pp. 81-85, 2011.

[25] P. Kyosti, et al., WINNER II channel models, WINNER II Public Deliverable, Sept. 2007.

[26] A. F. Molisch, Wireless Communications, 2nd edition. IEEE Wiley, 2011.

[27] Y.-P. Zhang and Y. Hwang, "Enhancement of rectangular tunnel waveguide model, in Microwave Conference Proc., 1997. APMC 97, 1997 Asia-Pacific, vol. 1, pp. 197C200, 1997.

[28] C. Briso, J. Cruz, and J. Alonso, "Measurements and modeling of distributed antenna systems in railway tunnels, IEEE Trans. Veh. Technol., vol. 56, no. 5, pp. 2870C2879, 2007.

[29] K. Guan, Z. Zhong, B. Ai, and C. Briso, "Novel Hybrid Propagation
Model inside Tunnels," IEEE 75th Veh. Technol. Conf. (VTC2012Spring), Yokohama, Japan, pp.1-5, May 2012.

[30] Z. Xu and $\mathrm{H}$. Zheng, "The effect of wall roughness on the electromagnetic wave propagation in coal mine underground," in Proc. IEEE Int. Symp. Knowl. Acq. Model., pp. 482C485, 2008.

[31] R. Martelly and R. Janaswamy, "Modeling radio transmission loss in curved, branched and rough-walled tunnels with the ADI-PE method, IEEE Trans. Antennas Propag., vol. 58, no. 6, pp. 2037C2045, Jun. 2010.

[32] F. Fuschini, G. Falciasecca, "A Mixed RaysłModes Approach to the Propagation in Real Road and Railway Tunnels", IEEE Trans. Antennas and Propag, vol. 60, no.2, pp. 1095-1105, Feb. 2012. 